\title{
Intracellular Photoactivation and Quantification Using Fluorescence Microscopy: Chemical Tools and Imaging Approaches
}

\author{
Giovanni Bassolino and Pablo Rivera-Fuentes*
}

\begin{abstract}
Recent advances in optical microscopy enable the visualization and quantification of biological processes within live cells. To a great extent, these imaging techniques remain limited by the physical properties of the chemical probes that are used as fluorescent tags, detectors and actuators. At the same time, the quantification of concentrations in the intracellular medium is not trivial, but a few approaches that employ optical microscopy have been developed. Herein, we highlight a few examples of how a combination of novel chemical probes and microscopy methods could be used to bring a much-needed quantitative dimension to the field of biological imaging.
\end{abstract}

Keywords: Fluorescence microscopy · Fluorophores · Imaging · Photoactivation · Uncaging

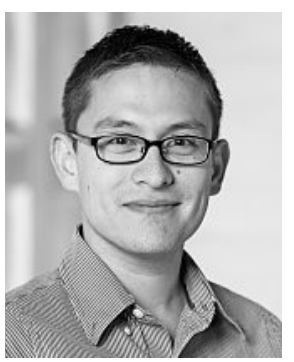

Institute of Molecular Medicine), he joined the Laboratory of Organic Chemistry at ETH Zurich as Assistant Professor (2015). Research in his group focuses on developing molecular probes to detect and manipulate small molecules in live cells.

\section{Introduction}

Optical microscopy has contributed

Pablo Rivera-Fuentes was born in 1984 in Mexico City and received a BSc (2008) degree in chemical engineering from the National Autonomous University of Mexico. He subsequently received MSc (2009) and PhD (2012) degrees in chemistry from ETH Zurich, working under the supervision of Prof. François Diederich. His $\mathrm{PhD}$ work was awarded the ETH Medal in 2013. Funded by the Swiss National Science Foundation (SNSF), he carried out postdoctoral research at Massachusetts Institute of Technology with Prof. Stephen J. Lippard (20122014). After a short postdoctoral stint at the University of Oxford with Prof. Harry L. Anderson (Department of Chemistry) and Prof. Christian Eggeling (Weatherall

${ }^{\star}$ Correspondence: Prof. Dr. P. Rivera-Fuentes Laboratorium für Organische Chemie ETH Zürich, $\mathrm{HCl}$ G329

Vladimir-Prelog-Weg 3, CH-8093 Zürich

E-mail: pablo.rivera-fuentes@org.chem.ethz.ch strategies, but rather we showcase a selection of those that highlight the benefits of combining novel synthetic probes with quantitative microscopic and bioanalytical techniques.

\section{Chemical Tools}

Photoactivatable molecules, also known as 'caged' or 'photocaged', constitute a class of molecular probes that allow the activation of a bioactive molecule using light (Scheme 1a). This activation mode has the advantage of providing excellent spatial and temporal resolution. ${ }^{[3]}$ The general concept of photoactivatable probes is that the protecting group masks the biological activity of the molecule of interest until it is cleaved through a photochemical reaction. This strategy has been applied to a very diverse range of biologically active species, including metal ions, ${ }^{[4,5]}$ diatomic signaling agents (i.e. $\mathrm{NO}, \mathrm{CO}$, etc.), ${ }^{[5]}$ neurotransmitters, ${ }^{[6-8]}$ lipids, ${ }^{[9]}$ nucleic acids, ${ }^{[8]}$ sugars, ${ }^{[3,8]}$ and proteins. ${ }^{[8]}$ Whereas light has been the most popular trigger to activate chemical probes, ${ }^{[3]}$ other stimuli have been successfully applied, including enzymatic cleavage ${ }^{[10]}$ and bioorthogonal reactions with small molecules. ${ }^{[11,12]}$

Many photoremovable groups have been applied to the activation of fluorophores. ${ }^{[13,14]}$ One reason is that it is experimentally convenient to evaluate the properties of new photoremovable groups when the released molecule becomes colored and/or fluorescent. In addition, photoactivatable fluorophores possess other 


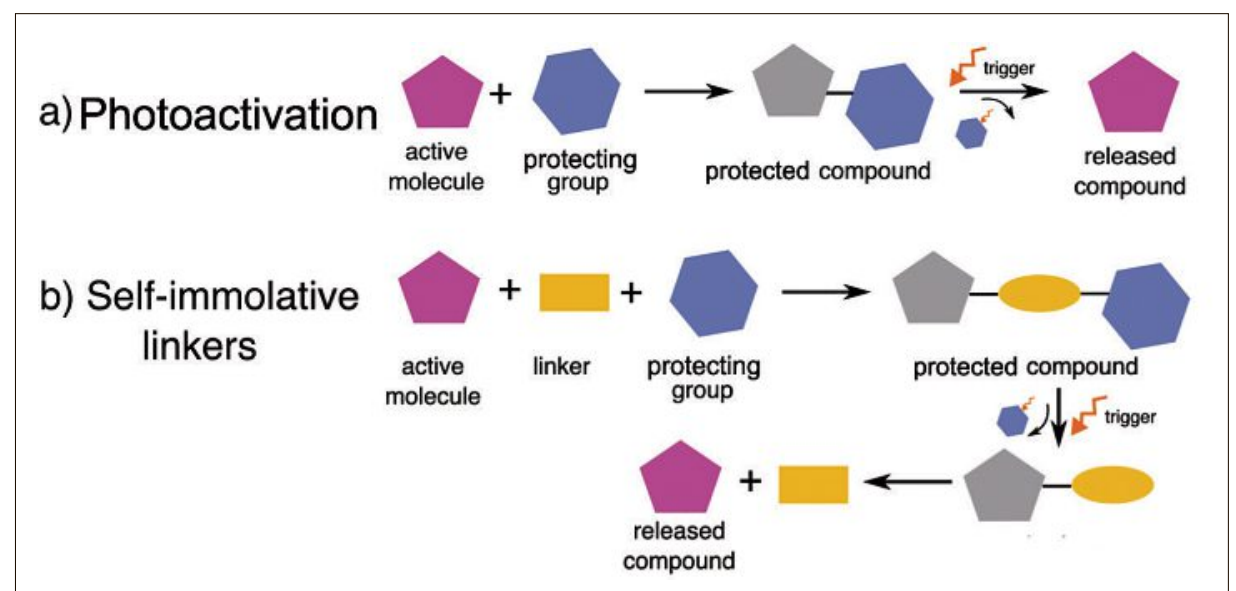

Scheme 1. Chemical tools and principles useful for controlled release in quantitative fluorescence microscopy. a) Photoactivatable compounds and b) self-immolative linkers. A biologically active molecule or a fluorophore (represented as a magenta pentagon) is rendered temporarily inactive or non-fluorescent (grey pentagon) through covalent binding with a protecting group (blue hexagon). Interaction with an external stimulus (orange lightning) cleaves the protecting group, resulting in the activation of the protected compound. When self-immolative linkers are used, upon removal of the protecting group, the linker itself undergoes spontaneous rearrangement (symbolized as a change in shape) in order to release the active molecule.

interesting applications. For instance, emitters that can be turned on with light are useful for super-resolution microscopy, in particular in localization microscopies. ${ }^{[15]}$ Photoactivatable dyes are also useful for labeling and tracking cells, ${ }^{[16]}$ to evaluate exchange rates of intercellular junctions ${ }^{[17]}$ or to calibrate photolysis experiments. ${ }^{[18]}$

A less explored area is the development of protecting groups that become fluorescent (fluorogenic) or change their emission wavelength after they are cleaved from the biologically active molecule. The development of such protecting groups is challenging because the functional groups that facilitate photoactivation are often incompatible with high quantum yields of luminescence (e.g. $\mathrm{NO}_{2}$ groups). ${ }^{[19]}$ A strategy that has overcome these difficulties is to insert a spacer that, upon release from the protecting group, undergoes a spontaneous rearrangement resulting in the release of the bioactive molecule of interest and a fluorescent molecule (Scheme 1b). ${ }^{[20,21]}$ These reactive spacers were introduced in the 1980 s $^{[21]}$ and are known as 'selfimmolative' linkers because they trigger their own elimination. ${ }^{[22]}$ A number of selfimmolative linkers has been developed, mostly based on benzylic eliminations or spontaneous intramolecular cyclization following the release from the protecting group. [22-24]

Among the probes that exploit selfimmolative linkers and other rearrangements, the three shown in Scheme 2 stand out because, upon release of the molecule of interest, they generate a fluorescent species in a 1:1 stoichiometry. These examples include coumarin derivatives $\mathbf{1}$ and $\mathbf{2}$, and cyanine dye $\mathbf{3}$. This class of protecting group is of particular interest for quantita- tive microscopy because the stoichiometric release of a fluorescent reporter makes it possible to quantify the amount of released (bio-)molecule.

Coumarin $\mathbf{1}$ is based on the elimination of a leaving group from a benzyliclike position promoted by the increased electron-donating ability of the deprotected hydroxide group. This self-immolative linker has been incorporated into a series of enzyme-activated compounds (i.e. compound 4, Fig. 1) and used as reporter in drug delivery systems by Shabat and coworkers. ${ }^{[25]}$ The compounds were tested in live cells, and it was observed that more intense fluorescence correlated with an increase in released drug, as determined by HPLC analysis of cell lysates. Although quantitative microscopic approaches could have been used to determine the intracellular concentration in individual cells, no such quantification was included in the report. ${ }^{[25]}$ It is worth highlighting that although HPLC analysis of lysates gives useful information about the population of cells, it does not account for cell-to-cell variability. In particular when studying complex populations of cells, such as tissue or whole animals, being able to obtain quantitative information directly from imaging data will greatly increase our ability to understand biological systems.

Linker 3, also developed by Shabat and coworkers, operates based on an orthobenzylic elimination. This linker was combined with a hydrogen peroxide-activated trigger to generate probe 5 (Fig. 1), which was used for selective drug delivery of anticancer agents to mice. ${ }^{[26]}$ Similarly to the coumarin linker, a correlation between fluorescence intensity and amount of drug released was measured in vitro. The modular nature of these compounds makes them attractive candidates for the development of light-activated, self-immolative compounds that could potentially be applied in quantitative microscopy.

The Porter ${ }^{[27]}$ and Jullien groups ${ }^{[28,29]}$ developed a conceptually different fluorogenic, photoremovable protecting group. These trans-cinnamic acid derivatives release the molecule of interest triggered by a photoinduced cis-trans isomerization and subsequent intramolecular lactonization (Scheme 2b). ${ }^{[27]}$ In particular, compound 6 (Fig. 1) has the advantage of being efficiently activated under two-photon excitation conditions, ${ }^{[28,29]}$ which is desirable because light of longer wavelength displays deeper penetration into non-translucent media. Exploiting the large contrast in fluorescence between the protected (transcinnamic acid) and activated (coumarin) product, the photoinduced release of cytosolic ethanol from the cinnamic ester in zebrafish embryos was quantified via fluorescence correlation spectroscopy. ${ }^{[28,29]}$

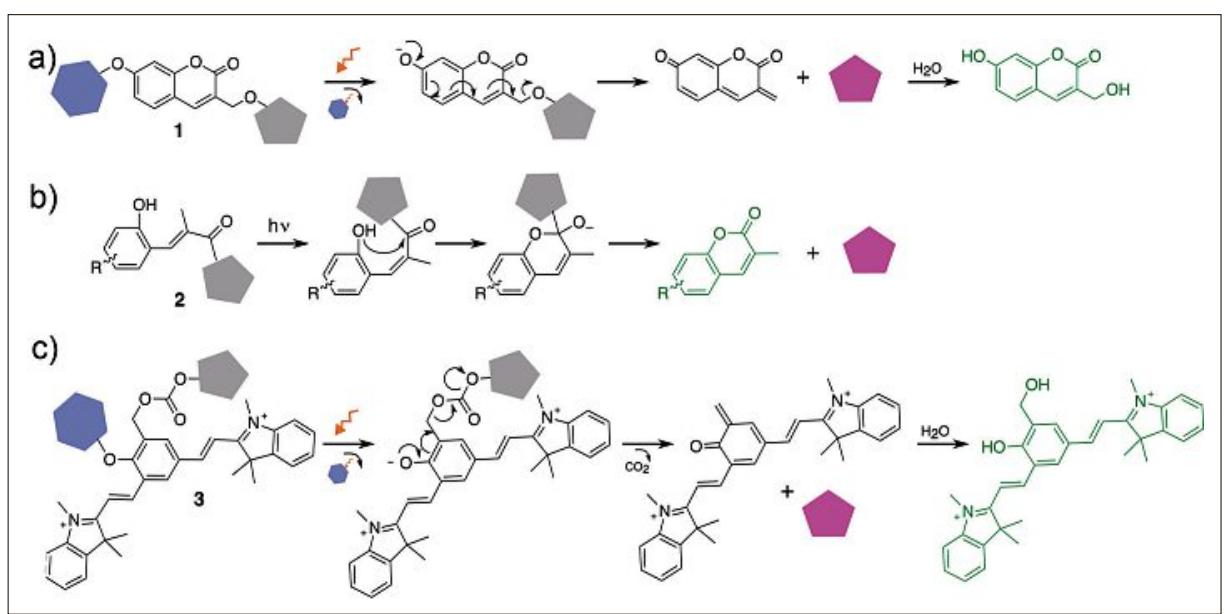

Scheme 2. Examples of fluorogenic, small-molecule releasing units (1-3) and their mechanisms of release. The structures of the released fluorophores are shown in green.

Hexagon = protecting group; pentagon = activatable molecule. 


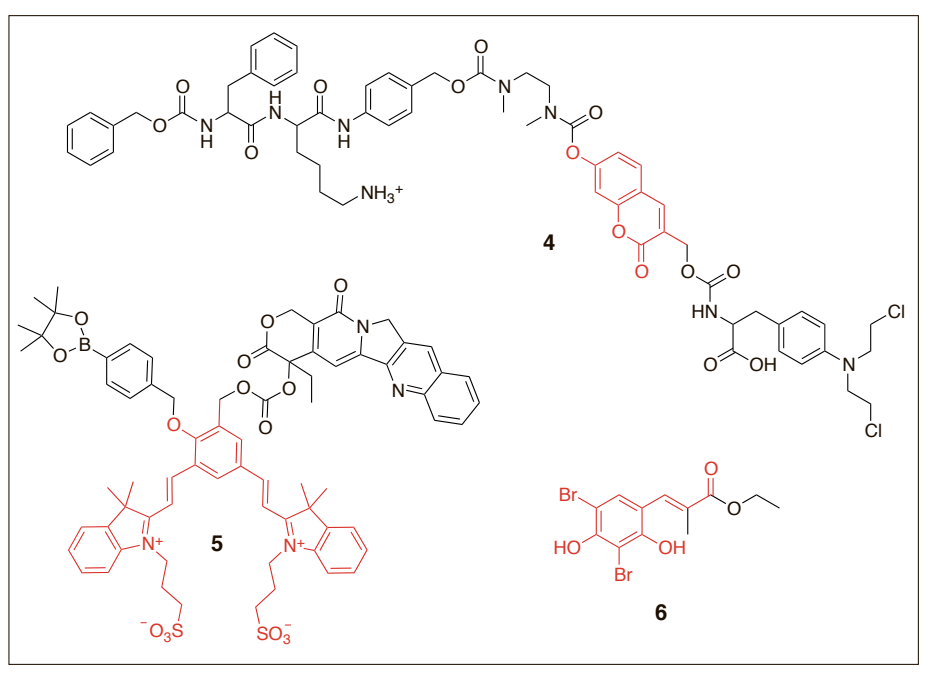

Fig. 1. Compounds used for the fluorogenic release of small molecules. The fluorogenic unit is colored red.

This study is a good example of how to photoactivate a bioactive molecule and quantify its concentration directly using microscopic techniques.

\section{Approaches for Quantifying the Absolute Concentrations of Dyes Using Microscopy}

In general, the concentration of a dye in a biological sample cannot be inferred directly from the intensity of its emission recorded by fluorescence microscopy. The reason is that the emission quantum yields of small-molecule emitters can vary greatly depending on their concentration, the $\mathrm{pH}$ of the medium, the presence of quenchers, the polarity, and even the temperature and viscosity of the sample. As a consequence, more information about the photophysical properties of the dye and the conditions of the medium are needed for quantitative microscopy.

A technique that can provide some of this information is fluorescence correlation spectroscopy (FCS). In FCS, the fluctuations in the fluorescence of a small ensemble of equilibrated molecules are recorded. ${ }^{[30-32]}$ The local concentration of molecules can be extracted with the aid of computational modeling provided that an autocorrelation trace of the fluorescence intensity is available. ${ }^{[30-32]}$ An autocorrelation function is built by measuring the fluctuations of the intensity against the mean value over time and reports on how the values correlate as a function of their separation within the time series (i.e. the time interval between signals). ${ }^{[33]}$ In order to obtain meaningful autocorrelation curves, only very few emitters need to be measured at once, but this difficulty is overcome in a confocal setup because the illuminated volume can be decreased to the order of femtoliters by tight focusing of the laser beam. ${ }^{[31]}$ Models are available to describe the autocorrelation function in terms of the diffusion time constants and the number of molecules within the illuminated volume, for populations of both single and multiple emitters. ${ }^{[30,32]}$ In the case of compound $\mathbf{6}$, there is a large contrast between the dark cinnamic ester and the activated, highly emissive coumarin, and therefore it is safe to assume that the only emitting species is the latter. In this way, the authors could estimate an initial intracellular concentration of the protected compound, which was very close to the one used to incubate the sample $(0.5-10 \mu \mathrm{M})$. Another example of quantification in live cells via FCS is the work by Cluzel and coworkers, who quantified the expression of the chemotactic signaling protein Che-Y fused to GFP in order to characterize the behavior of individual flagellar motors in E. coli. ${ }^{[34]}$

Alternatively, the construction of calibration curves has been suggested as a method to relate the fluorescence intensity observed in a confocal microscope to the concentration of the dye of interest in the sample. ${ }^{[35]}$ This method is based on the assumption that the fluorescence intensity measured toward the center of an object larger than the point spread function generated by the microscope optics is directly proportional to the fluorophore concentration, provided that the region probed by the focused beam is entirely contained within the cellular volume.[35] Following this approach, Loew and coworkers built a calibration curve by measuring the fluorescence intensity of a series of solutions of different concentrations of the dye (Fura-2, a calcium indicator) between coverslips, keeping the same instrumental parameters used for cell imaging. A similar approach was followed by Schultz and coworkers to quantify bioactive lipids photoreleased from coumarin-protected derivatives ${ }^{[36]}$ and by Hsu and Dervan to quantify polyamide-fluorescein conjugates in cellular nuclei. ${ }^{[37]}$ In both studies, calibration curves were built taking a series of images of standard solutions of known amounts of refer- ence dye and interpolating the measured fluorescence intensity of the sample.[36,37] Hsu and Dervan further validated their quantification by building a calibration curve in a flow cytometer with commercially available fluorescent standard beads. This method also involved the estimation of the concentration by interpolation into a calibration curve. Even though there were some discrepancies between the results obtained by each method, the measured concentration was consistently lower than in the incubating solution used, ${ }^{[37]}$ giving valuable information about the membrane permeability of their polyamides.

Fluorescent standard beads are microspheres with different loading levels of one or multiple dyes. ${ }^{[38,39]}$ The use of such beads for calibration is based on the concept of 'molecules of equivalent soluble fluorophore' (MESF). ${ }^{[40]}$ To assign an MESF value to a population of fluorescent beads, a calibration curve is built by plotting the fluorescence intensity against concentration for a series of solutions of the same dye bound to the beads. The fluorescence intensity of a suspension with a known number of beads is measured, and the equivalent number of fluorophores in solution is assigned by interpolation employing the calibration curve. Assignment of a MESF value to a fluorescent bead does not require the emission quantum yield of the dye in the bead and in solution to be the same or even known. MESF values are assigned by comparison of the absorbance-normalized fluorescent intensities that, under the appropriate experimental conditions, are dependent on the product of the number of molecules present and the quantum yield of emission, a procedure that does not require knowledge of either parameter for the sample to be characterized. ${ }^{[40]}$ On the other hand, the dependence of the emission quantum yield on the environment matters when using the beads to estimate the absolute number of fluorophores in an unknown sample (e.g. the intracellular environment), ${ }^{[38,39]}$ because the quantum yield of emission for the dye in the sample might well be different from the one in the standard solutions used to generate the original MESF calibration curve. In fact, the dependence of the dye spectral features on the environment is a problem that all these methods with external calibration share, and it highlights the importance of developing new fluorescent dyes with emission that has minimal sensitivity towards the environment.

Chiu and coworkers used an alternative approach to estimate the number of emitters per imaged region. In their studies, they fitted the measured intensity distribution for small ensembles of labeled proteins with the intensity distribution of a single labeled protein. ${ }^{[41,42]}$ The starting 
point was to obtain a set of intensities from fluorescent regions where there is only a single protein and find the distribution that best describes the set (usually a lognormal one). That signal was then used as the basis for fitting the distribution intensities of regions where more proteins are present. They found that these intensities are not linearly related (i.e. the intensities of a region with 10 proteins is not 10 times the intensity of the individual one). Instead, they estimated the number of emitters by finding the best fit for the intensity distribution by multiplying the distribution of the individual labeled protein by an integer. ${ }^{[41,42]}$ The lognormal character of the distribution arises from instrumental factors that are function only of the position within the image but not the fluorophore concentration; they also considered factors related to the labeling efficiency, such as the existence of a preferential labeling site, quenching of the fluorophore by the antibody or different extent of labeling between proteins. In the system under study, the authors found that these factors do not affect the lognormal character of the distribution within the experimental and fit errors. ${ }^{[42]}$ Both studies were performed on proteins that were labeled using organic fluorophores, therefore it is possible that this approach could be used for small-molecule fluorophores in solution, but this hypothesis remains to be tested.

\section{Closing Remarks}

With the advent of advanced imaging techniques and improved equipment, the time is ripe to start using optical microscopy as a widespread quantitative technique. The development of new fluorogenic molecules specifically tailored to take advantage of this opportunity is pivotal and demonstrates that chemistry is at the heart of modern microscopy and bioimaging. In this review we focused on selected strategies that could be applied to the activation and quantification of small molecules in the intracellular medium. This is one of the goals of our research group at ETH Zurich, and our current work is directed towards being able to release small molecules, in quantifiable amounts, within selected subcellular compartments using fluorescence microscopy. A combination of synthetic chemistry, chemical biology, and quantitative microscopy will be crucial for the development and application of these probes.

\section{Acknowledgements}

Work in our laboratory is funded by a start-up grant from ETH Zurich, the ETH Zurich Research Grant ETH-02 16-1 and the Swiss National Science Foundation grant 200021_165551.

Received: August 9, 2016

[1] V. C. Coffman, J.-Q. Wu, Trends Biochem. Sci. 2012, 37, 499.

[2] J. C. Waters, J. Cell Biol. 2009, 185, 1135.

[3] P. Klán, T. Šolomek, C. G. Bochet, A. Blanc, R. Givens, M. Rubina, V. Popik, A. Kostikov, J. Wirz, Chem. Rev. 2013, 113, 119.

[4] C. Gwizdala, S. C. Burdette, in 'Inorganic Chemical Biology: Principles, Techniques and Applications', Ed. G. Gasser, John Wiley \& Sons, Ltd., Chichester, U.K., 2014, p. 275.

[5] K. L. Haas, K. J. Franz, Chem. Rev. 2009, 109, 4921.

[6] V. R. Shembekar, Y. Chen, B. K. Carpenter, G. P. Hess, Biochemistry 2007, 46, 5479.

[7] N. Senda, A. Momotake, T. Arai, Bull. Chem. Soc. Jpn. 2007, 80, 2384

[8] G. Mayer, A. Heckel, Angew. Chem. Int. Ed. 2006, $45,4900$.

[9] D. Höglinger, A. Nadler, C. Schultz, Biochim. Biophys. Acta - Mol. Cell Biol. Lipids 2014, 1841,1085

[10] C. Ritter, N. Nett, C. G. Acevedo-Rocha, R. Lonsdale, K. Kräling, F. Dempwolff, S. Hoebenreich, P. L. Graumann, M. T. Reetz, E. Meggers, Angew. Chem. Int. Ed. 2015, 54, 13440.

[11] J. Li, P. R. Chen, Nat. Chem. Biol. 2016, 12, 129.

[12] J. Kim, C. R. Bertozzi, Angew. Chem. Int. Ed. 2015, 54, 15777.

[13] L. M. Wysocki, J. B. Grimm, A. N. Tkachuk, T. A. Brown, E. Betzig, L. D. Lavis, Angew. Chem. Int. Ed. 2011, 50, 11206.

[14] F. M. Raymo, ISRN Phys. Chem. 2012, 619251.

[15] F. M. Raymo, J. Phys. Chem. Lett. 2012, 3, 2379.

[16] D. J. Kozlowski, E. S. Weinberg, Methods Mol. Biol. 2000, 135, 349 .

[17] W.-h. Li, G. Zheng, Photochem. Photobiol. Sci. 2012, 11, 460.

[18] G. P. Ahern, S.-F. Hsu, M. B. Jackson, J. Physiol. 1999, 520, 165.

[19] B. Valeur, M. N. Berberan-Santos, in 'Molecular Fluorescence: Principles and Applications', Wiley-VCH Verlag $\mathrm{GmbH} \&$ Co. KGaA, Weinheim, Germany, 2012, p. 75.
[20] P. K. Chakravarty, P. L. Carl, M. J. Weber, J. A. Katzenellenbogen, J. Med. Chem. 1983, 26, 638.

[21] P. L. Carl, P. K. Chakravarty, J. A. Katzenellenbogen, J. Med. Chem. 1981, 24, 479.

[22] A. Alouane, R. Labruère, T. Le Saux, F Schmidt, L. Jullien, Angew. Chem. Int. Ed. 2015, 54, 7492.

[23] M. E. Roth, O. Green, S. Gnaim, D. Shabat, Chem. Rev. 2016, 116, 1309.

[24] S. Gnaim, D. Shabat, Acc. Chem. Res. 2014, 47 , 2970.

[25] R. Weinstain, E. Segal, R. Satchi-Fainaro, D Shabat, Chem. Commun. 2010, 46, 553 .

[26] O. Redy-Keisar, S. Ferber, R. Satchi-Fainaro, D. Shabat, ChemMedChem 2015, 10, 999.

[27] A. D. Turner, S. V. Pizzo, G. Rozakis, N. A. Porter, J. Am. Chem. Soc. 1988, 110, 244.

[28] N. Gagey, P. Neveu, C. Benbrahim, B. Goetz, I. Aujard, J.-B. Baudin, L. Jullien, J. Am. Chem. Soc. 2007, 129, 9986.

[29] N. Gagey, P. Neveu, L. Jullien, Angew. Chem. Int. Ed. 2007, 46, 2467.

[30] O. Krichevsky, G. Bonnet, Rep. Prog. Phys. 2002, 65, 251 .

[31] P. Schwille, Cell Biochem. Biophys. 2001, 34, 383.

[32] S. A. Kim, K. G. Heinze, P. Schwille, Nat. Methods 2007, 4, 963.

[33] G. E. P. Box, G. M. Jenkins, G. C. Reinsel, in "Time Series Analysis", John Wiley \& Sons, Inc., Hoboken, NJ, 2013, pp. 21.

[34] P. Cluzel, M. Surette, S. Leibler, Science 2000 , 287, 1652.

[35] C. Fink, F. Morgan, L. M. Loew, Biophys. J. 1998, 75,1648

[36] A. Nadler, D. A. Yushchenko, R. Müller, F Stein, S. Feng, C. Mulle, M. Carta, C. Schultz, Nat. Commun. 2015, 6, 10056.

[37] C. F. Hsu, P. B. Dervan, Bioorg. Med. Chem. Lett. 2008, 18, 5851 .

[38] Spherotech, Inc., 'SPHERO ${ }^{\mathrm{TM}}$ Calibration Particles'. The technical document can be found under http://www.spherotech.com/2016 Catalog Product Detail Pages/Spherotech Rainbow Calibration Beads for Cytometry Performance Verification.pdf, accessed July 27, 2016.

[39] Bangs Laboratories, Inc., 'Quantum ${ }^{\mathrm{TM}}$ MESF Kits'. The technical document can be found under http://www.bangslabs.com/sites/default/ files/imce/docs/PDS 821 Web.pdf, accessed July 27, 2016.

[40] A. Schwartz, L. Wang, E. Early, A. Gaigalas, Y.z. Zhang, G. E. Marti, R. F. Vogt, J. Res. Natl. Inst. Stand. Technol. 2002, 107, 83.

[41] S. A. Mutch, B. S. Fujimoto, C. L. Kuyper, J. S. Kuo, S. M. Bajjalieh, D. T. Chiu, Biophys. J. 2007, 92, 2926.

[42] S. A. Mutch, P. Kensel-Hammes, J. C. Gadd, B. S. Fujimoto, R. W. Allen, P. G. Schiro, R. M. Lorenz, C. L. Kuyper, J. S. Kuo, S. M. Bajjalieh, D. T. Chiu, J. Neurosci. 2011, 31, 1461. 